\title{
Word class and context affect alpha-band oscillatory dynamics in an older population
}

\author{
Monika S. Mellem ${ }^{1}{ }^{*}$, Marcel C. M. Bastiaansen ${ }^{2,3}$, Lea K. Pilgrim ${ }^{4}$, Andrei V. Medvedev $^{1}$ and \\ Rhonda B. Friedman ${ }^{1}$
}

${ }^{1}$ Department of Neurology, Georgetown University, Washington, DC, USA

${ }^{2}$ Max Planck Institute for Psycholinguistics, Nijmegen, Netherlands

${ }^{3}$ Donders Institute for Brain, Cognition and Behaviour, Radboud University, Nijmegen, Netherlands

${ }^{4}$ Department of Psychology, University of Stirling, Stirling, Scotland

\section{Edited by:}

Lucia Melloni, Max Planck Institute for Brain Research, Germany

\section{Reviewed by:}

Matti Laine, Abo Akademi University, Finland

Satu Palva, University of Helsinki,

Finland

\section{${ }^{*}$ Correspondence}

Monika S. Mellem, Department of Neurology, Georgetown University Medical Center, 4000 Reservoir Road, NW, Building D, Room 207, Washington, DC 20007, USA

e-mail:monikamellem@gmail.com
Differences in the oscillatory EEG dynamics of reading open class (OC) and closed class (CC) words have previously been found (Bastiaansen et al., 2005) and are thought to reflect differences in lexical-semantic content between these word classes. In particular, the theta-band $(4-7 \mathrm{~Hz})$ seems to play a prominent role in lexical-semantic retrieval. We tested whether this theta effect is robust in an older population of subjects. Additionally, we examined how the context of a word can modulate the oscillatory dynamics underlying retrieval for the two different classes of words. Older participants (mean age 55) read words presented in either syntactically correct sentences or in a scrambled order ("scrambled sentence") while their EEG was recorded. We performed time-frequency analysis to examine how power varied based on the context or class of the word. We observed larger power decreases in the alpha (8-12 Hz) band between 200-700 ms for the OC compared to $\mathrm{CC}$ words, but this was true only for the scrambled sentence context. We did not observe differences in theta power between these conditions. Context exerted an effect on the alpha and low beta $(13-18 \mathrm{~Hz})$ bands between 0 and $700 \mathrm{~ms}$. These results suggest that the previously observed word class effects on theta power changes in a younger participant sample do not seem to be a robust effect in this older population. Though this is an indirect comparison between studies, it may suggest the existence of aging effects on word retrieval dynamics for different populations. Additionally, the interaction between word class and context suggests that word retrieval mechanisms interact with sentence-level comprehension mechanisms in the alpha-band.

Keywords: lexical-semantic, reading, EEG, oscillatory dynamics, alpha, theta

\section{INTRODUCTION}

Understanding the myriad of operations underlying language comprehension in the brain is an ongoing challenge in the field. Neuronal networks supporting these operations must dynamically assemble locally and flexibly communicate across long distances. But how does the brain do this? A growing number of studies on the oscillatory dynamics during language processing are revealing new insights into these mechanisms (for a review, see Bastiaansen and Hagoort, 2006). Oscillatory synchronization is thought to reflect the transient formation of functional brain networks (Varela et al., 2001; Fries, 2005), and this synchronization can be measured through EEG or MEG spectral analysis. Power analysis is thought to reflect the recruitment of local neuronal networks while coherence analysis reflects dynamic linking between brain areas involved in a global network. Additionally, analyzing oscillatory dynamics may reveal a rich set of information that is not currently seen during event-related potential (ERP) analysis because the ERP tends to average out non-phase-locked signals. Thus the study of oscillatory dynamics is a promising method to complement current ERP analysis and examine functional networks for language processing.

The network dynamics of lexical-semantic processing are still not fully understood. Previous studies have indicated that theta oscillations $(4-7 \mathrm{~Hz})$ are involved in these networks for lexicalsemantic retrieval. Bastiaansen et al. (2005) observed greater theta power increases between 300 and $500 \mathrm{~ms}$ when subjects read open class (OC) words compared to closed class (CC) words in a short story context. Since OC words carry more semantic information than CC words, they are thought to probe lexical-semantic retrieval processing more deeply than CC words. In addition, these authors observed greater alpha $(8-12 \mathrm{~Hz})$ power decreases between 300 and $500 \mathrm{~ms}$ at right occipitotemporal electrodes for OC than CC words. Thus alpha power decreases may also be related to semantic retrieval. A study comparing nouns with different semantic features (primarily visual vs. auditory) also found differences in theta power increases between 100 and $400 \mathrm{~ms}$ (Bastiaansen et al., 2008). The theta effect was larger for the "visual" nouns at occipital electrodes while it was larger at temporal electrodes for "auditory" nouns suggesting that theta scalp topography 
reflects the sensory features of the words. Both studies point to a role for theta oscillations in the retrieval of lexical-semantic information, but the robustness of this effect has yet to be fully established.

In the present study, we focus on the role of oscillatory dynamics in retrieving OC vs. CC words. Specifically, we want to investigate the robustness of the previously established theta and alpha effects in different contexts and in a different population. Our motivation stems from how the factors of context and age affect the well-studied N400 ERP component. The N400 is a negative-going potential between 300 and $500 \mathrm{~ms}$ that reflects lexical-semantic processing. Evidence shows that the N400 is modulated by both the class (e.g., Van Petten and Kutas, 1991; Nobre and McCarthy, 1994; King and Kutas, 1995; Münte et al., 2001) and context of words (e.g., Kutas and Hillyard, 1980, 1984). An interaction between word class and context has also been reflected in the N400 (Van Petten and Kutas, 1991). Thus we have chosen to experimentally manipulate both word class (OC, CC) and context (sentence, scrambled sentence) in this study to further investigate any interaction between the two factors on the oscillatory dynamics during word reading. Aging also affects the amplitude and latency of the N400 (Harbin et al., 1984; Gunter et al., 1992; Kutas and Iragui, 1998), and could potentially modulate oscillatory responses. So far, the oscillatory dynamics of lexical-semantic processing have not been investigated in an older population (Bastiaansen et al., 2005 tested younger participants, aged 2128) though the changes in the N400 suggest that there may be changes in the underlying semantic processing mechanisms with age. Hence we have chosen to test in an older population. An additional aim is to examine gamma band $(>30 \mathrm{~Hz})$ dynamics. This will extend the previous study on OC and CC words (Bastiaansen et al., 2005) which did not examine gamma oscillations though prior research has suggested that it is involved in lexical-semantic processing (Pulvermüller et al., 1999; Mainy et al., 2008).

To summarize, we will be testing for word class and context effects in older subjects as well as for any interaction between these factors. We predict that modulation of theta and alpha power will reflect greater lexical-semantic processing for OC words than CC words. Additionally, we expect that an interaction between class and context will reflect how word-level and sentence-level processing interact.

\section{MATERIALS AND METHODS SUBJECTS}

Fourteen healthy volunteers participated in the experiment and received monetary compensation for their participation. Two were excluded from the analysis due to excessive eye movements. Two additional subjects were excluded due to missing or corrupted files. Thus analyses were performed on data from 10 healthy subjects (mean age 55, age range 44-77, five males). All were right-handed, native English speakers without a history of neurological disorders. None were on medications that would have affected their performance during this study. All gave informed consent (approved by the Georgetown University Medical Center Institutional Review Board) before starting the experiment.

\section{STIMULI}

Fifty-six sentences were created to include as wide a variety of $\mathrm{CC}$ words as possible. The number of words per sentence ranged from 10 to 20 with an average of 15 . The sentences were derived from 163 unique OC words (e.g., nouns, verbs, and adjectives) and 69 unique CC words (e.g., pronouns, prepositions, and conjunctions). After repetitions of these words, sentences contained a total of 863 words, but a subset of 645 critical words (CW) was selected for analysis. This allowed us to exclude first and last words to avoid start-up and wrap-up effects as well as any words that fit into both open and closed categories. Additionally, each of the 56 sentences was turned into a scrambled sentence, e.g., the sentence "the girl sat on the floor" became "sat floor the girl on the." Thus each CW occurred both in a sentence context and in a scrambled sentence context. There were $640 \mathrm{CWs}$ in the set of scrambled sentences (a few had to be removed post hoc); thus subjects read a total of 1285 CWs. Among the total set of CWs, we examined 695 OC words and $590 \mathrm{CC}$ words.

The selected OC words ranged in length from 2 to 13 characters and had a log frequency of $0-3.66$ (Kucera-Francis log frequency per million words). The selected CC words ranged in length from 1 to 15 characters and had a frequency of $0.30-4.84$. The average length of OC vs. CC words differed significantly [OC: 5.78 and CC: $3.57 ; t(643)=14.29, p<0.001]$; similarly, there were significant differences in the log frequency [OC: 2.05 and CC: 3.68; $t(643)=-23.98, p<0.001]$.

\section{DESIGN AND PROCEDURE}

We used a $2 \times 2$ within-subjects factorial design with factors context (sentence or scrambled sentence) and word class (OC or CC). Subjects were instructed to silently read words presented on a CRT computer monitor and respond to comprehension questions spoken by the experimenter after each trial. The words appeared in white lower-case letters on black background in Arial font. The words subtended a horizontal visual angle of at most $4.8^{\circ}$ (for the 15-letter word). The subjects were asked to minimize any movements and eyeblinking while words were on the screen. The schematic (Figure 1) shows the general organization of the paradigm and task. Stimuli were presented in four blocks of sentences alternating with four blocks of scrambled sentences per session (out of two sessions). Each sentence block contained 14 trials of sentences while each scrambled sentence block contained 14 trials of scrambled sentences; thus in each session there were 112 trials. Within a trial, words were displayed one at a time at the center of the screen for $400 \mathrm{~ms}$ with an ISI of $400 \mathrm{~ms}$ (stimulus onset asynchrony was $800 \mathrm{~ms}$ ) until the end of the sentence or scrambled sentence. Trials were also repeated with a word ISI of $800 \mathrm{~ms}$ (randomly interspersed with $400 \mathrm{~ms}$ trials), but these trials are not examined here. For half of the sentences, the sentence context was presented first. For the other half of the sentences, the scrambled sentence context was presented first; thus, the order of context (sentence vs. scramble sentence) was counterbalanced.

Between each trial, subjects answered questions to confirm that they were reading the words. The questions varied based on the type of trial: for example, "Was this sentence about a circus?" for a sentence or "Did you see the word 'circus'?" for a scrambled sentence. The experimenter manually recorded the responses. Average 


\section{Sentence Block}

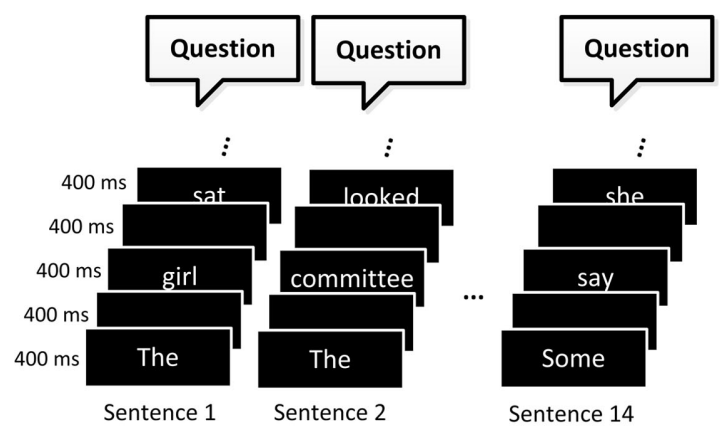

Scrambled Sentence Block

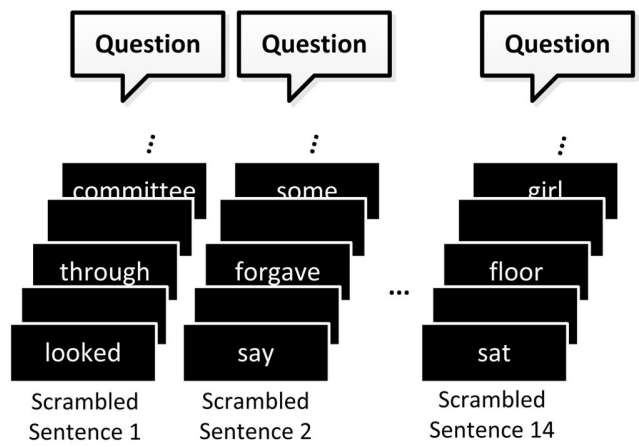

FIGURE 1 | Schematic representing the general organization of the paradigm and the task. Alternating blocks of sentences and scrambled sentences (order counterbalanced across subjects) were presented to the subjects. Each sentence block contained 14 trials accuracy was $86 \%$ for the sentence questions and $70 \%$ for the scrambled sentence questions which indicates an overall attentiveness to the readings. Data from both correct and incorrect trials were subject to preprocessing and analysis. The inter-trial interval varied based on response time of the subject to the question. While reaction time was not collected, subjects typically responded within several seconds. All subjects saw all words in both contexts across two sessions separated by 1 week. Each session was under $2 \mathrm{~h}$, including time for preparation, recording, and cleanup.

\section{EEG RECORDING AND PREPROCESSING}

Scalp EEG was continuously recorded from 64 tin electrodes mounted in an elastic cap (Electro-cap International, USA). Electrodes were also placed above and below the right eye and on the outer canthi of both eyes to record vertical and horizontal eye movements and blinks. The signals were referenced to the left mastoid and sampled at a rate of $500 \mathrm{~Hz}$. Impedance at each electrode was kept at or below $5 \mathrm{k} \Omega$. The signals were amplified by Neuroscan SynAmps 2 amplifiers (Neuroscan, USA) and filtered on-line with a band-pass filter $(0.05-100 \mathrm{~Hz}, 24-\mathrm{dB} /$ octave attenuation). Data were stored off-line for further analysis.

For all preprocessing and subsequent analysis, we used the FieldTrip toolbox (Oostenveld et al., 2011) which is implemented in Matlab (Mathworks, MA, USA). We performed the following preprocessing steps: re-referencing to the average of the left and right mastoid channels, band-pass filtering $0.3-100,60 \mathrm{~Hz}$ line filtering, and artifact removal. Prior to artifact removal, EEG epochs were extracted from the continuous EEG from $1000 \mathrm{~ms}$ before, to $2000 \mathrm{~ms}$ after onset of the CWs. Single word epochs with artifacts that occurred between $200 \mathrm{~ms}$ before to $800 \mathrm{~ms}$ after stimulus onset were removed from analysis. A $Z$-score based algorithm was used to detect eye blinks and eye movements, and other artifacts were detected with a threshold algorithm. After removal, about $70 \%$ of single word epochs remained. There were an average (marginal means) of 436 single word epochs in the sentence condition, 462 in the scrambled sentence condition, 480 in the OC condition, and 417 in the CC condition. The cell means were 220 for OC in sentence, 260 for OC in scrambled sentence, 216 for CC of sentences while each scrambled sentence block contained 14 trials of scrambled sentences. The experimenter asked the subject questions after each trial to ensure subject comprehension and attention.

in sentence, and 202 for CC in scrambled sentence. A two-way ANOVA on these groups did not reveal statistical differences in the remaining number of words between these conditions.

\section{TIME-FREQUENCY ANALYSIS}

In order to reveal event-related changes in power for the different frequency components of the EEG, time-frequency representations (TFR) of the single word trial data were computed by using the multi-taper approach described by Mitra and Pesaran (1999). TFRs were constructed in two different, partially overlapping frequency ranges. In the low-frequency range $(2-30 \mathrm{~Hz})$, 2.5-Hz frequency-smoothing and $400 \mathrm{~ms}$ time-smoothing Hanning windows were used to compute power changes in frequency steps of $1 \mathrm{~Hz}$ and time steps of $10 \mathrm{~ms}$. In the high-frequency range $(25-100 \mathrm{~Hz})$, power changes were computed in $2.5-\mathrm{Hz}$ frequency steps and $10 \mathrm{~ms}$ time steps, with $2.5-\mathrm{Hz}$ frequency-smoothing and $400 \mathrm{~ms}$ time-smoothing discrete prolate spheroidal sequence windows. These calculations were performed starting $500 \mathrm{~ms}$ before the word appeared on the screen to $1500 \mathrm{~ms}$ after the word onset. We calculated TFRs in two different ways to examine both evoked and induced activity, i.e., oscillatory activity that is both phaselocked and non-phase-locked to the stimuli. For the first method, we calculated the TFRs on individual trials and averaged across those trials; this analysis reflects both induced and evoked activity. For the second method, we calculated the ERP by averaging over trials and then calculated the TFRs; this analysis reflects evoked activity only. By performing both types of analysis, we were able to determine if any effects were due to evoked or induced activity.

The TFRs of single word epochs were averaged across the following four conditions: words in a sentence, words in a scrambled sentence, OC words, CC words. To calculate the event-related power changes, the TFRs were expressed as percent increases or decreases relative to a 200 -ms pre-stimulus baseline.

\section{STATISTICAL ANALYSIS}

The significance of the difference between each two pairs of conditions was evaluated by means of a cluster-based random permutation test (Maris and Oostenveld, 2007). This approach controls the 
Type-1 error rate in a situation involving multiple comparisons. The cluster-based random permutation test naturally takes care of interactions between time points, electrodes, and frequency bins by identifying clusters of significant differences between conditions in the time, space, and frequency dimensions. The procedure is briefly described below.

First, for every data point (electrode by time by frequency) of two conditions, a simple dependent-samples $t$-test is performed (giving uncorrected $p$-values). All adjacent data points exceeding a preset significance level (5\%) are grouped into clusters. For each cluster the sum of the $t$-statistics is used in the cluster-level test statistic. Next, a null distribution which assumes no difference between conditions is created. This distribution is obtained by 500 times randomly assigning the conditions in subjects and calculating the largest cluster-level statistic for each randomization. Finally, the actually observed cluster-level test statistics are compared against the null distribution, and clusters falling in the highest or lowest 2.5 th percentile are considered significant.

We examined the main effects of context and class by performing the pairwise comparisons sentence-scrambled sentence and OC-CC, respectively. Then we tested for an interaction between class and context using the permutation test as follows: first we did two subtractions (1) OC in scrambled sentence-CC in scrambled sentence and (2) OC in sentence-CC in sentence. Then we tested whether these subtractions are different (i.e., whether there is an interaction between the two factors) by performing a permutation test on these two subtractions. The resulting $p$-value pertains to the interaction effect. For each contrast, we performed the cluster analysis separately on lower frequencies $(4-30 \mathrm{~Hz})$ and higher frequencies $(25-100 \mathrm{~Hz})$.

\section{RESULTS}

The evoked analysis only revealed activity centered at $150 \mathrm{~ms}$ in the alpha-band. Additionally, we did not find any significant effects in the evoked responses for either the OC-CC comparison (Figure 2A) or the sentence-scrambled sentence (S-SS) comparison (Figure 2B). The significant effects presented below thus reflect induced activity.

\section{MAIN EFFECT OF CLASS}

The contrast OC-CC revealed a significant main effect of class in the alpha range (Figure 3). A cluster spanning 200-700 ms was present in the analysis $(p=0.046)$. This effect resulted from a greater power decrease for OC words than for CC words. This was a widespread effect strongest over right occipital and left frontal areas. We examined but did not find a significant cluster in the gamma range.

\section{MAIN EFFECT OF CONTEXT}

The contrast sentence-scrambled sentence revealed a significant cluster spanning $0-700 \mathrm{~ms}$ in the alpha and low beta range ( $p=0.006$; Figure 4). The overall effect indicated by this single negative cluster resulted from less power in the sentence condition than the scrambled sentence condition, specifically, an early smaller increase followed by a greater decrease. The cluster spanned both anterior and posterior areas. We examined but did not find a significant cluster in the gamma range.

\section{INTERACTION EFFECT}

The interaction effect was marginally significant $(p=0.08)$ for lower frequencies; however, since establishing a possible interaction between context and word class was one of the central issues behind this work, we also tested the simple effects. We found a significant cluster for the OC-CC contrast in the scrambled sentence context $(p=0.016)$ which was centered on the alpha range between 150 and $700 \mathrm{~ms}$ (Figure 5). We did not find a significant cluster for the $\mathrm{OC}-\mathrm{CC}$ contrast in the sentence context.

\section{DISCUSSION}

In order to examine the role of oscillatory dynamics during lexicalsemantic processing in an older population, we studied changes in power as subjects read OC and CC words in different contexts. Our results indicate that alpha $(8-12 \mathrm{~Hz})$ oscillations are differentially involved in reading words of different class, while both alpha and low beta $(13-18 \mathrm{~Hz})$ are implicated in reading words in different contexts. To follow-up on the previous study of OC and CC words (Bastiaansen et al., 2005), we also examined how context can affect reading words of different classes.

The main finding of the study was a greater power decrease in the alpha-band for OC than CC words; this effect spanned 200$600 \mathrm{~ms}$ over left frontal and right posterior areas. While power increases in the theta and gamma bands typically reflect active processing, the opposite is true for the alpha frequency range. Here, power decreases are generally thought to reflect more cortical processing or engagement (e.g., Jokisch and Jensen, 2007; Klimesch et al., 2007). As OC words carry more lexical-semantics than CC words, reading OC words involves more lexical-semantic retrieval. Thus our results support the notion that alpha power decreases may be related to lexical-semantic retrieval operations. Evidence for this relationship comes from several other studies. In their comparison of OC vs. CC words, Bastiaansen et al. (2005) found not only theta power increases but also significant alpha power decreases between 300 and $500 \mathrm{~ms}$ at right posterior channels. The topography of our alpha effect looks remarkably similar to the topography found in their study. This suggests that alpha reflects similar processes in both studies. Studies of semantic judgment tasks have further implicated the involvement of alpha power decreases with semantic processing (Klimesch et al., 1997; Röhm et al., 2001; reviewed in Klimesch, 1999). Evidence from several studies also suggests that alpha power decreases can reflect increased attention (see Klimesch, 1999 for a review). It is unlikely that there were greater attentional demands in reading OC words compared with CC words; thus we favor a semantic interpretation for the word class effect.

Given the consistency in the alpha response across studies, it was notable that we did not observe a significant effect in the theta range. The main results from the motivating studies (Bastiaansen et al., 2005, 2008) pointed to theta power increases as involved in lexical-semantic retrieval in a younger population of subjects. In contrast, our data suggest that theta power may not play a role in lexical-semantic processing in an older population. Instead, for older participants, semantic processing mechanisms may rely more on alpha oscillatory dynamics. We want to emphasize that the comparison of these effects across the two different studies is indirect and qualitative at best. Our study cannot speak directly 
A Evoked Data, Contrast OC - CC
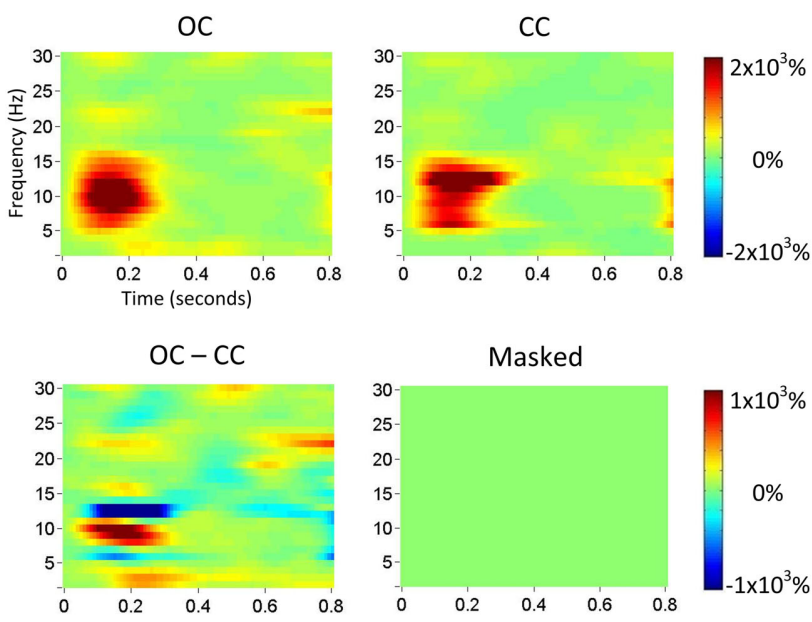

B Evoked Data, Contrast Sentence - Scrambled Sentence
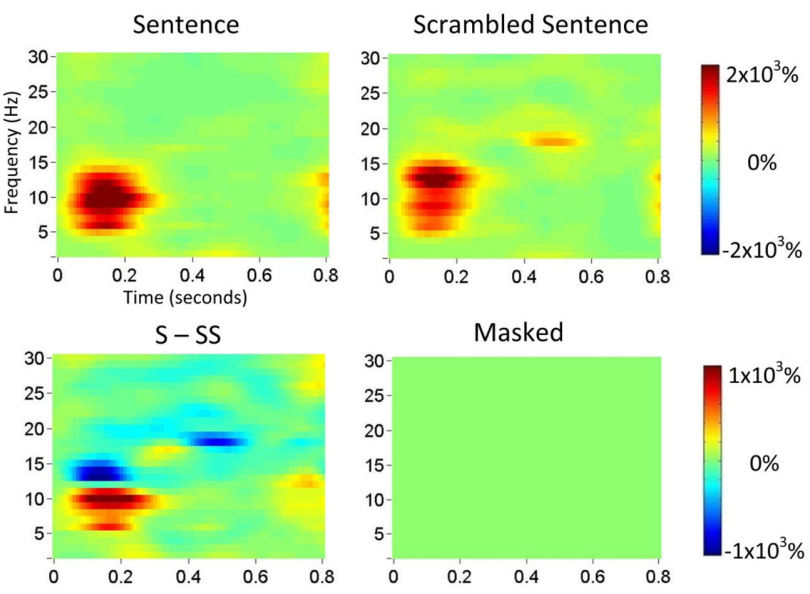

FIGURE 2 |Time-frequency representations (TFRs) of evoked data.

(A) The evoked responses for open class (OC) and closed class (CC) conditions at a left frontal channel (F3). TFRs are shown separately for the OC and $\mathrm{CC}$ conditions, the raw difference between these conditions (OC-CC), and the statistically thresholded difference (Masked). Plots are shown as a relative change compared to baseline. (B) The evoked responses for sentence (S) and scrambled sentence (SS) conditions at a left frontal channel (FC3). No significant difference exists for either comparison. to aging effects in the theta and alpha-bands but rather suggests the existence of aging effects, a suggestion that should be further investigated by a direct comparison between two age groups in a subsequent study. Still a comparison between the studies is warranted as they used similar stimuli (open and closed class words in a sentence context presented at the same duration and rate as our study) and tasks (participants were instructed to read the words and were asked comprehension questions). Two main differences were the inclusion of a second variable in our study, that of context (Bastiaansen et al., 2005 did not present words outside of a sentence context), and the languages of presentation (Dutch vs. English). There is no evidence that oscillatory dynamics differ based on the testing language.

Previous evidence suggests that theta power may decrease with age. Studies of working memory have observed that theta power in older subjects decreases relative to younger subjects (Karrasch et al., 2004; Cummins and Finnigan, 2007). Thus it is possible that we are observing an effect of aging in the shift from theta-band effects to alpha-band effects though this cannot be said with more confidence without younger controls. Ultimately, the previously established theta power increase does not appear to be a robust phenomenon related to lexical-semantic retrieval in all populations. Further work directly comparing these different populations within the same study would lend more support to the hypothesis that decreases in theta power during lexical-semantic processing are related to aging.

The observation that alpha and beta power was smaller for words in a sentence than a scrambled sentence between 0 and $700 \mathrm{~ms}$ suggests that context modulates the oscillatory dynamics in these bands. A methodological challenge exists, however, in interpreting this context effect. Since our time-frequency analysis computed all power changes relative to pre-word baselines, the post-word differences observed in the sentence-scrambled sentence contrast may have two possible causes: (1) the processingrelated differences of these words, and (2) the processing-related differences in the baseline periods before each open or closed 

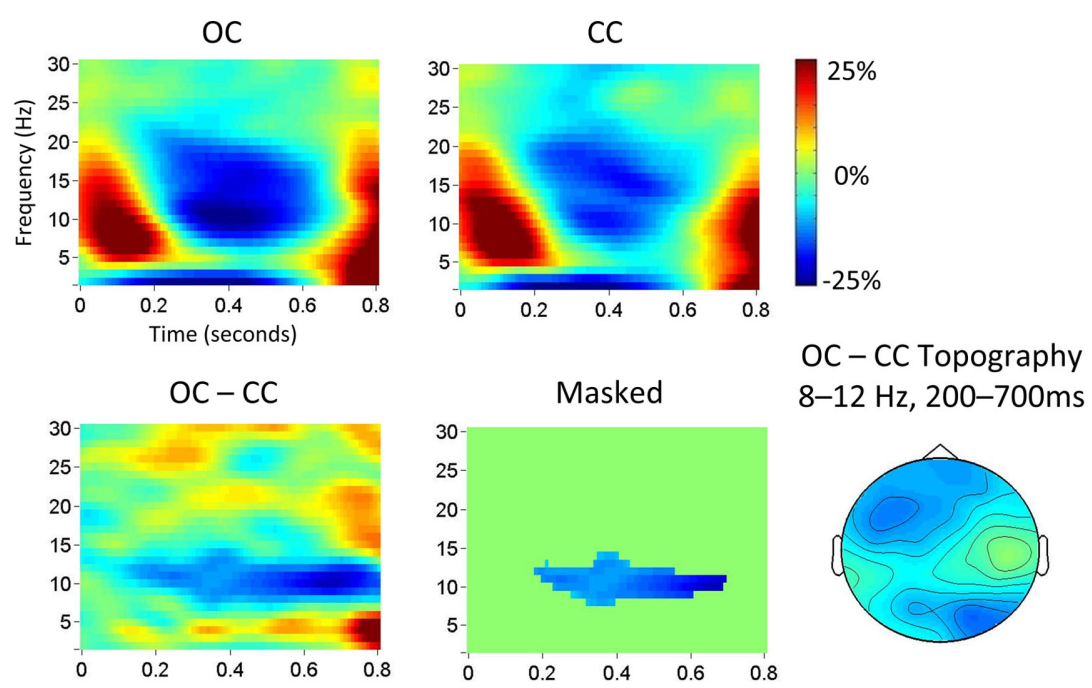

FIGURE 3 |Time-frequency representations showing the alpha-band difference between the $O C$ and $C C$ conditions at a left frontal channel

(F3). The topography shows the raw difference between conditions during the time of the significant alpha effect. See legend to Figure $\mathbf{2}$ for more details.
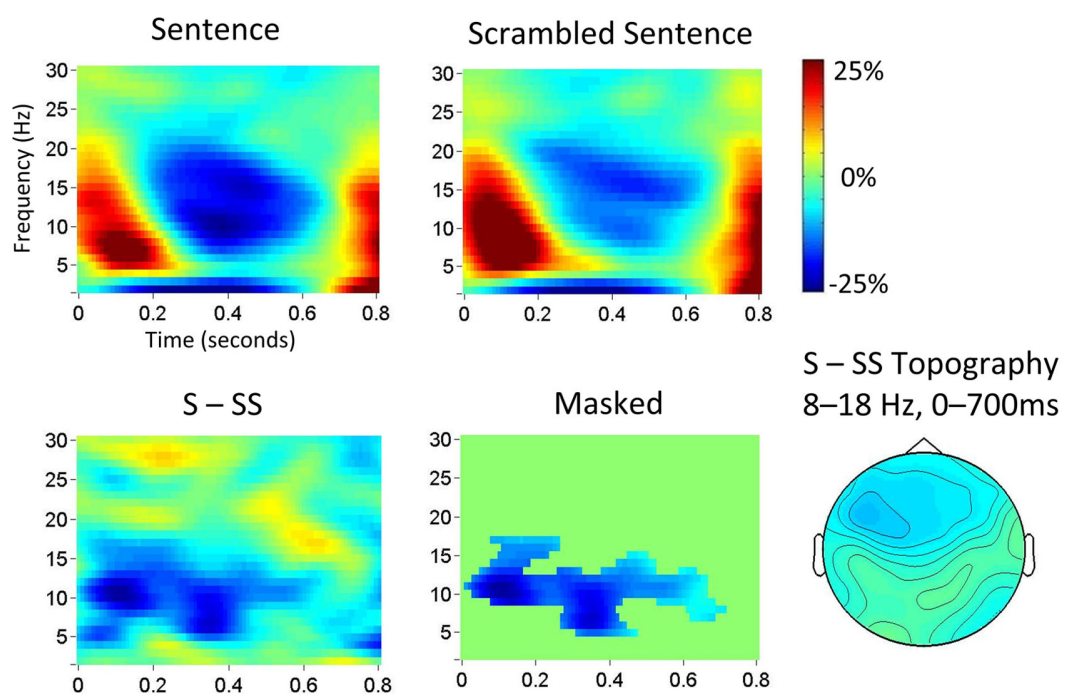

FUR 4 | Time-frequency representations showing the alpha- and low beta-band difference between the sentence and scrambled sentence conditions at a left frontal channel (FC3). See legend to Figure $\mathbf{2}$ for details.

class word. The reason for these baseline differences is that the baseline power in the sentence context is likely to be influenced by sentence-level processes such as syntactic parsing, semantic integration, working memory demands, or even attentional demands. These processes are probably not (or at least to a lesser extent) engaged in the scrambled sentence context which leads to the possible differences in baseline power between sentence and scrambled sentence conditions. Therefore, as this comparison was not ideally controlled, we would feel it misleading to interpret the alpha and beta power changes as specifically reflecting one of these sentence-level processes. Further work with more tightly controlled conditions could help disambiguate the observed context main effects.
The previous study of OC and CC words by Bastiaansen et al. (2005) only included words within a sentence context; thus it was not clear if the observed effects solely reflected word-level processing or may include sentence-level processing also. In order to examine how reading words of different class may be affected by context, we inspected the interaction between these factors. We followed up the marginally significant interaction with simple effects tests of OC-CC in the scrambled sentence context and OC-CC in the sentence context and only found a significant effect for the contrast in the scrambled sentence context. Thus the class effect observed in the scrambled sentence context does not show up in the sentence context. This might indicate that the main effect of class is driven mainly by words in the scrambled sentence context 
A Contrast OC - CC in a Scrambled Sentence Context
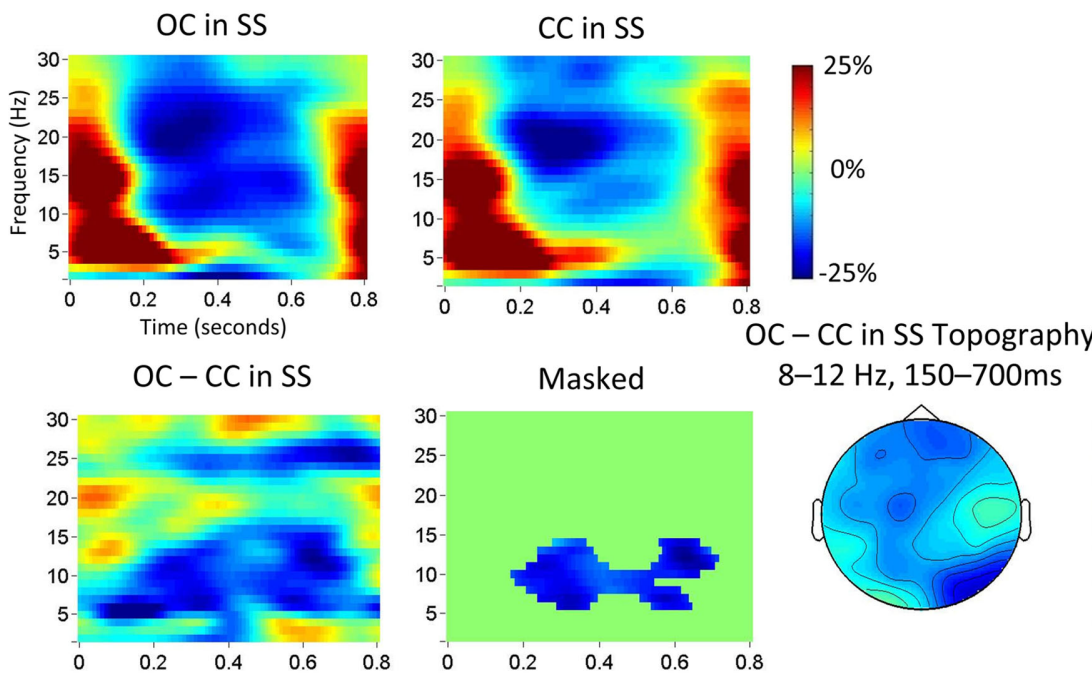

8-12 Hz, 150-700ms

B Contrast $\mathrm{OC}-\mathrm{CC}$ in a Sentence Context
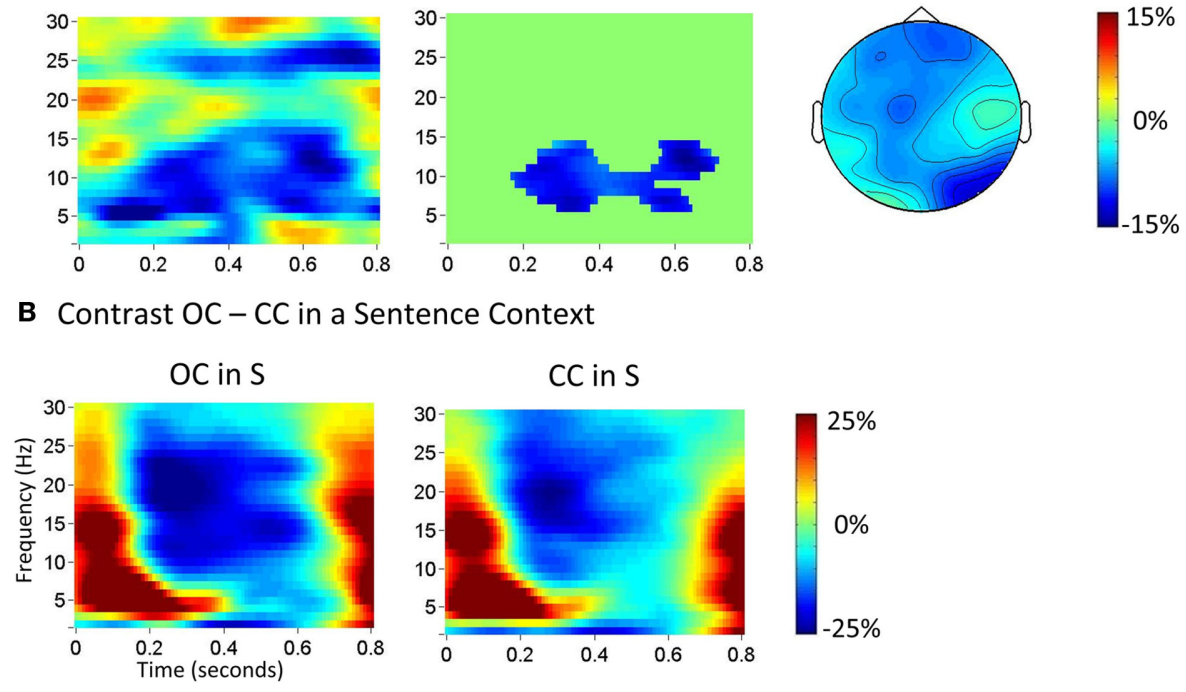

$\mathrm{OC}-\mathrm{CC}$ in $\mathrm{S}$

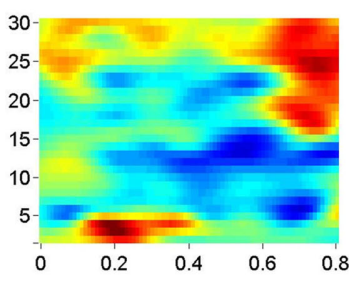

Masked

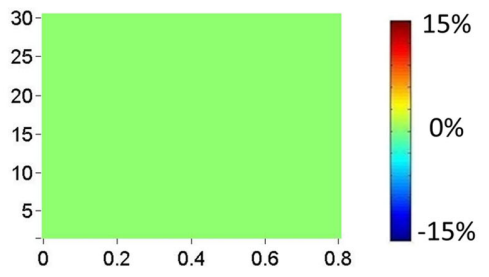

FIGURE 5 |TFRs showing (A) the alpha-band difference between the OC and CC conditions in the scrambled sentence (SS) context at a right posterior channel (O2), and (B) no significant difference between the OC and CC conditions in the sentence (S) context. See legend to Figure 2 for details.

and therefore primarily reflects word retrieval mechanisms. But alpha power decreases are present in both main effects of word class and context which suggests that alpha is driven by both word retrieval and sentence processing. We tentatively propose that an interaction between word class and context arises because the class effect is being obscured by sentence-level processing such that it is not seen in the sentence context. As mentioned above, alpha power decreases have been previously linked to attentional processing (see Klimesch, 1999 for a review). Thus one possibility is that larger attention-related alpha decreases in the sentence context may make it difficult to reliably detect the additional alpha decreases due to word class differences, at least not as clearly as observed in the scrambled sentence context. Thus greater attentional allocation during sentence reading than during scrambled sentence reading may be "swamping" the class effect.
Additionally, we wanted to extend the previous study on OC vs. CC words (Bastiaansen et al., 2005) by examining potential effects in the gamma frequency band (i.e., above $30 \mathrm{~Hz}$ ). However, we did not observe significant gamma power changes between $\mathrm{OC}$ and CC words. Since a relationship between gamma power increases and sentence-level semantic unification/integration has previously been observed (Hagoort et al., 2004; Hald et al., 2006; Pena and Melloni, 2011), this absence of gamma band effects might suggest that processing lexical-semantics relies partly on different neuronal mechanisms from processing sentence-level semantics. The current literature suggests that the neuronal dynamics of lexicalsemantic processing may be confined to the lower frequencies, as opposed to previously observed sentence-level semantic processes which are situated in the gamma frequency range. However, more work is needed to further substantiate such a claim. 
Several potential confounds exist in this study. In comparing OC and CC words to examine differences in semantic content, it must be remembered that these word classes naturally have different frequencies and lengths. While we did see significant differences in both frequency and length, evidence is currently lacking that these variables affect oscillatory dynamics. Bastiaansen et al. (2005) did not find that word frequency influenced the amplitude of oscillatory responses. Thus, it is probably unlikely that any word frequency difference would influence our power results. Another potential confound is repetition effects as we had more repetitions for CC words than OC words. We did try to minimize this repetition and maximize exposure to a variety of $\mathrm{CC}$ words by including 69 unique CC words, but more repetition for CC words naturally reflects the higher word frequency of $\mathrm{CC}$ words. To our knowledge, there are no published reports of repetition effects on oscillatory dynamics during visual word processing with repetition lags greater than one (as we had here).

\section{REFERENCES}

Bastiaansen, M., and Hagoort, P. (2006). Oscillatory neuronal dynamics during language comprehension. Prog. Brain Res. 159, 179-196.

Bastiaansen, M. C. M., Oostenveld, R., Jensen, O., and Hagoort, P. (2008). I see what you mean: theta power increases are involved in the retrieval of lexical semantic information. Brain Lang. 106, 15-28.

Bastiaansen, M. C. M., van der Linden, M., Ter Keurs, M., Dijkstra, T., and Hagoort, P. (2005). Theta responses are involved in lexicalsemantic retrieval during language processing. J. Cogn. Neurosci. 17, 530-541.

Cummins, T. D. R., and Finnigan, S. (2007). Theta power is reduced in healthy cognitive aging. Int. J. Psychophysiol. 66, 10-17.

Fries, P. (2005). A mechanism for cognitive dynamics: neuronal communication through neuronal coherence. Trends Cogn. Sci. (Regul. Ed.) 9, 474-480.

Gunter, T. C., Jackson, J. L., and Mulder, G. (1992). An electrophysiological study of semantic processing in young and middle-aged academics. Psychophysiology 29, 38-54.

Hagoort, P., Hald, L., Bastiaansen, M., and Petersson, K. M. (2004). Integration of word meaning and world knowledge in language comprehension. Science 304, 438-441.

Hald, L. A., Bastiaansen, M. C., and Hagoort, P. (2006). EEG theta and gamma responses to semantic violations in online sentence processing. Brain Lang. 96, 90-105.

Harbin, T., Marsh, G., and Harvey, M. (1984). Differences in the late components of the eventrelated potential due to age and to

\section{CONCLUSION}

We observed a word class effect only in the alpha-band in a sample of older subjects. Thus the previously observed word class effects on theta power changes in a younger participant sample do not seem to be a robust effect across populations of different age. This suggests that there may be different neuronal dynamics during word retrieval for different populations. Moreover, the interaction between word class and context suggests that there is an interaction of word retrieval mechanisms with sentence-level comprehension mechanisms in the alpha-band.

\section{ACKNOWLEDGMENTS}

This work was partially supported by NIDCD R01 DC007169 and R01 HD036019 (Rhonda B. Friedman and Lea K. Pilgrim), NIDCD F31DC010545 (Monika S. Mellem), and NCRR R21RR025786 (Andrei V. Medvedev). Preparation of the manuscript was partially supported by NIDCD R01 DC10780.

task across 6 decades. Electroencephalogr. Clin. Neurophysiol. 108, 456-471.

Mainy, N., Jung, J., Baciu, M., Kahane, P., Schoendorff, B., Minotti, L., Hoffmann, D., Bertrand, O., and Lachaux, J. P. (2008). Cortical dynamics of word recognition. Hum. Brain Mapp. 29, 1215-1230.

Maris, E., and Oostenveld, R. (2007). Nonparametric statistical testing of EEG- and MEG-data. J. Neurosci. Methods 164, 177-190.

Mitra, P. P., and Pesaran, B. (1999). Analysis of dynamic brain imaging data. Biophys. J. 76, 691-708.

Münte, T. F., Wieringa, B. M., Weyerts, H., Szentkuti, A., Matzke, M., and Johannes, S. (2001). Differences in brain potentials to open and closed class words: class and frequency effects. Neuropsychologia 39, 91-102.

Nobre, A. C., and McCarthy, G. (1994). Language related ERPs - scalp distributions and modulation by word type and semantic priming. J. Cogn. Neurosci. 6, 233-255.

Oostenveld, R., Fries, P., Maris, E., and Schoffelen, J. M. (2011). Fieldtrip: open source software for advanced analysis of MEG, EEG, and invasive electrophysiological data. Comput. Intell Neurosci. 2011, 1-9.

Pena, M., and Melloni, L. (2011). Brain oscillations during spoken sentence processing. J. Cogn. Neurosci. 11491164. doi:10.1162/jocn_a_00144. [Advanced Access published Oct. 7, 2011].

Pulvermüller, F., Lutzenberger, W., and Preissl, H. (1999). Nouns and verbs in the intact brain: evidence from event-related potentials and highfrequency cortical responses. Cereb. Cortex 9, 497-506.
Röhm, D., Klimesch, W., Haider, H., and Doppelmayr, M. (2001). The role of theta and alpha oscillations for language comprehension in the human electroencephalogram. $\mathrm{Neu}$ rosci. Lett. 310, 137-140.

Van Petten, C., and Kutas, M. (1991) Influences of semantic and syntactic context on open- and closedclass words. Mem. Cognit. 19, 95-112.

Varela, F., Lachaux, J. P., Rodriguez, E., and Martinerie, J. (2001). The brainweb: phase synchronization and large-scale integration. Nat. Rev. Neurosci. 2, 229-239.

Conflict of Interest Statement: The authors declare that the research was conducted in the absence of any commercial or financial relationships that could be construed as a potential conflict of interest.

Received: 20 December 2011; accepted: 14 March 2012; published online: 09 April 2012.

Citation: Mellem MS, Bastiaansen MCM, Pilgrim LK, Medvedev AV and Friedman RB (2012) Word class and context affect alpha-band oscillatory dynamics in an older population. Front. Psychology 3:97. doi: 10.3389/fpsyg.2012.00097

This article was submitted to Frontiers in Language Sciences, a specialty of Frontiers in Psychology.

Copyright (C) 2012 Mellem, Bastiaansen, Pilgrim, Medvedev and Friedman. This is an open-access article distributed under the terms of the Creative Commons Attribution Non Commercial License, which permits non-commercial use, distribution, and reproduction in other forums, provided the original authors and source are credited. 